worm is always typical in its aspect, which, of course, paves the way for the neglect of careful observation. The third is; in my opinion, a belief founded on imperfect observation, that ringworm generally leaves behind a certain amount of scurfiness after the real disease is cured. I demur to the truth of this last proposition. If microscopic exsmiastion be made in such cases of apparent scurfiness only, it will be found that the disease, as evidenced by the presence of diseased hairs and fungus, still exists, but not in an ative state, though in certain cases where the scalp has been mach inflamed and irritated, simple desquamation may have gone on for a time. Microscopic examination alone can decide this point. For all practical purposes scurfuess should be regarded as indicative of disease still present. It is a question of taking a little trouble to make a proper examination, and as any mistake on the point is liable to be followed by serious consequences, if a child be pronounced well when it is not, such trouble ought to be taken.

As regards the confusion of ringworm with eczema, it is to be wembered that eczema usually affects not only the head. but adjoining parts where its true nature is reoognised; that eorema, although it causes a matting together and, it may be, general thinning of the hair, is never attended by the production of such a morbid, dry, brittle state of the hairs as is found in ringworm. Where these are found, even if ecrema is also present, the treatment is that of riag worm.

segards seborrboa, the absence in it of all traces of diseased hairs is the sure guide to a correct diagnosis where there is doubt.

There is, lastly, an exceptional state that should be men. tioned liable to be misunderstood, and that is overtreated singworm. I have had cases of disease brought to ne in wiob undoubted ringworm has existed, but in which the disease has been cared, and the surface kept in a state of imritation by the still further and free use of irritating parasiticides. Of course, in such instances the microscope discloses bealthy hair shafts and root sheaths, an absence of fungus and diseased hairs, and the presence only of pus and epitholial débris.

(To be continued.)

ON THE

VENOUS CIRCULATION IN RELATION TO SOME OF THE DISEASES WHICH AFEECT THE LOWER LIMB.

BY JOHN GAY, F.R.C.S.,

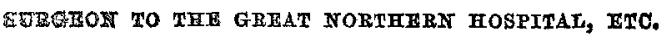

(Concluded from p. 42.)

DROPSY.

15e course of the following remarks on dropsy I shall assume, first, that in both the anasarcous and saccalar Gom of this disorder the fluid is derived from the venocispillary vessels of the fibrillar connective tissue, which with constitutes the chief ingredient of the subdermoid gtrucsures in one case, and, with a varying proportion of the same slement, the tissue of the serous sacs in the other; and, socondly, that with identity of texture the fluid is in both the setare-viz., water, holding in solution alkaline salts, and a sua or more, in different cases, of albumen. Adventitious elamonts are, of course, often found in this fluid, such as bisod, pis, fibrinous flakes, organised matter, and other pro. acts-innocent and malign-of morbid action incidental to the tissues in which it is found, especially the serous sacs, Wy wich it may become complex in the highest degree; but theae are in no wise concerned with the pathology of aimple dropsical or anasarcous effusion. They are but its somtingent complications. The dropsy to which the following remarks are designed to apply is the simple serous afision, and as such might be tabulated, as far as the nature the fuid is concerned, as tegumentary when seated in the divin-the so-called cdema or anasarca; and pericardiac, peritonitic, pleuritic, or other, as the case may be, when collected in serous sacs.

A distinction will, however, have to be kept in mind between the forms of tegumentary dropsy just alluded to, which have a clear pathological history, and those other varieties for which it has been difficult to assign an acceptable cause-viz., the renal, anæmic, and perhaps those which belong to some of the exanthems, carbuncle and the like.

It may be true equally of every form of dropsy that it is simply the result of an excess in the rate at which the fluid which naturally lubricates the connective tissues is effused above that at which it is absorbed; but there is no doubt that, in their production severally, other factors peculiar to each are at work, to which I must for a moment call atten. tion, in order to anticipate exceptions on this ground that may be taken to the views I am about to offer.

In renal and anæmic dropsy the blood is at fault; and although no reason has been given, as far as $I$ am aware, why it should thus part with a portion of its serum, the inspissidity of this element, or some other abnormal quality of the blood itself, may explain it in part, or, it may be, entirely. With regard to the exanthemata, however, the case differs ; for in these disorders the extent of the dropsy is usually coincident with that of the skin affection. And here, as I propose to show presently, the physiological anatomy of the capillary system comes to our aid; for it exhibits in the relations of its mucous and muscular elements to their common trunks and to each other an order of arrangement very similar to that which, as I shall show, obtains in the general venous system, in the conditional disturbance of which I venture to think the immediate raison d'etre of dropsical effusion is to be found.

Lymph circulation, according to the best authorities, see Recklinghausen on the Lymphatic System (Stricker), appears to be as follows: The rootlets of the lymphvessels are distributed through the tissues, and stand in connexion with the bloodvessels in such a manner that they can withdraw the serous fluid from them, and return it by their terminal trunks; the origin of the lymph-system being in direct continuity with the capillary vessels through certain intermediate or adjoining (ly mphatic) spaces which are the temporary receptacles of the fluid that exudes from them. The exudation is effected by the pressure under which the blood moves in the capillary vessels; whilst its absorption by the lymphatics is due to favouring blood-pressure or tension in that part of the vein-system in which they terminate, the chief venous trunks. Hence, as an excess of blood-tension in the trunk and systemic veins would be adverse to the reabsorption of the serum, effused for normal purposes, into the veins, it accumulates in the serous spaces and becomes dropsy. Physiologically, there is little doubt that dropsy is due to blood-tension in these veins from physical retardation of their currents.

But there is an apparent anomaly connected with this explanation which has scarcely received notice. It is thisthat dropsical effusions are limited to the connective tissues appertaining to those capillaries only of the tegumentary tissues and serous sacs which are anatomically and dynamically within the range of the obstructing agent. They do not invade adjoining muscular and their allied textures. Now in order to dropsy a tributary as well as its trunk must suffer coincident obstruction; and then it is limited to the peripheral capillary area of the tegumentary vein. Hence its seat is always in anatomical relation to that of its cause. Thus, in the lower limb, or elsewhere, it is not an uncommon thing to see cedema limited to a certain area after an attack of phlebitis; but in order to this, the trunk, as well as the tributary, vein, which receives the blood from the œdematous tissues, must be coincidently involved. Physiology gives a reason for such limited cedematous patches, to which I shall presently allude.

Lower and Hewson long ago showed that ligature of the inferior cava was followed by ascites, and that ligature of the jugular veins-not of one only - was required in order to produce anasarca in the head of a dog. Gulliver added valuable testimony in support of this view, not only by observations which he made, but by collateral and still abiding proof in some preparations which, I believe, are still to be found in the Museum of the Army Medical Department at Chatham. Moreover, whether the cedema be in the immediate vicinity of the obstructed vein, 
which causes it, as in the case of a carbuncle, or remote from it, as in the case of an codematous hand with axillary cancer, or leg with mitral disease, the two are physiologically and anatomically separated by the distance that intervenes between the vein, or portion of the vein system, in which the exciting cause exists, and its peripheral capillary area. This is undoubtedly a commonplace observation, but in obscure cases it may help us, for it is well to rememher that the seat of codema in its earlier stages is no indication of that of its cause; for although exudation must necessarily take place into every lymphatic space that lies within the range alluded to, it simply sinks to the lowest level by the force of gravitation, and gradually Hoods a limb, according to the seat and persistence of the obstructing cause. Locality is a matter of gravitation; extent, a question of amount. The same rule applies to saccular dropsy; and its application enables us to determine the exact seat of the obstruction to which the filling of any such sac is respectively due.

But why is venous obstruction followed by any such abnormal results at all? In the cases of the arteries, the compensatory arrangements of their branches are such as, under the like circumstances, to maintain the circulation in a state of complete efficiency. This is not so, however, with the veins. The compensatory provisions in these are not so perfect as in the arteries. Take the case of a ligature on the femoral vein on the cardiac side of the saphenous junction. In such a case the limb would become oodematnus, but its vit.lity would not thereby be destroyed. This might, however, follow slight additional interference with the vein currents. In fact, there is a remarkable limitation of anastomotic agency for compensatory purposes on the part of these vessels. Thus the anastomosis between the femoral tributaries and those of the gluteal, ischiatic, epigastric, and other veins, is insufficient to compensate for tbe loss of the transmitting powers of the femoral in the case under consideration; and, as is well known, the familiar large and bloated veins which course orer the abdominal walls in cases of ascites, although they may serve to maintain the circulation, cannot release the peritoneum from the effects of the portal obstruction. In cases of "white leg," too, I think I have had sufficient pathological proof wherewith to endorse the fact that the cedema doss not disappear until the femoral current is re. established through the removal or tunneling of the clot.

This fact has its explanation in valvular ageney, to which I have heretofore alluded, especially the wonderful com. bination of distributive and converging power which it exercises over the currents through the veins, by which each, in a state of bealth, has its own rarticular and more or less direct course awarded to it. Indeed, from the capillary rills to the systemic veins in which they issue, these streams are kept in enforced independency of each other, almost to isolation, subject only to the disturbing effects of the constantly varying degrees of blood-tension to which they are prone.

But to return. Admitting, in accordance with the foregoing observations, that dropsy is, in the first place, due to retardation of the blood in a systemic or trunk vein, and, in the second, to the special want of natural provision for compensation by the establishment of collateral channels, the difficulty presents itself: Why are the tegumentary tissues, and, in like manner, the serous sacs, exclusively the seat of the effasion? Why does it not invade the muscular or motor structures that are in juxtaposition with them, seeing that a certain capillary area appertaining to the latter is always included with a corresponding area belonging to the former, within the range of the disturbing influence? Can an explanation of this seeming paradox be given; and, if so, can it be made to apply to both forms of dropsy, the saccular as well as the tegumentary? The points of resemblance that have been suggested as existing between the tegumentary tissues and the pericardium seem to justify the expectation that others bearing on this point might, on inquiry, be found to exist.

I have repeatedly examined cedematous limbs in every degree of distension, and have failed to find an exception to the foregoing statement; and Dr. Peacock has informed me, with regard to the heart, that he has seen the pericardium in every phase of serous distension, but he has not in any case, to his recollection, seen the muscular texture of the heart in like manner and coincidently infiltrated. Recently
1 amputated a limb in which the tegumentary tissues were severely odematous; but, as I pointed out at the time, there was not the slightest infiltration of the subfascia? textures. I will not undertake to eay that such a complication is a pathological myth; but I do say that it is very unusual-almost so as to claim to be a rule of that class of which it is said, for the sake of cautious reservation, that there is "none without exceptions."

Some few years ago, for otber than the objects of the present paper, I made this experiment upon the vein circulation in the hind limbs of a dog.

The whole of the thigh was encircled by ligatures, so as completely to prevent the return of any blood from the distal portion of the limb, excluding the femoral arters. The blood was allowed to pour in to the limb until the flow ceased from repletion. A ligature was then put on the femoral. and the animal destroyed. Amongst the results was strikingly this-viz., that the tissuez of the skin were odematous in the highest degree, but the coema had not extended to the muscular or motor structures, or to other textures below the deep fascia. Indeed there was acarcely a sign of congestion, whilst in the former it was carried to its extreme of intensification.

In order, as far as possible, without the help of an almost impossible experiment, to ascertain how far, upor anatomical grounds, an inference might be drawn in favour of a like relation between the veins of the muscular heart and it serous sac, Mr. Pearson, at my request, injected both separately, and in alternate sequences as to order. Indeed, tbere was scarcely a sign of congestion in their teztures. whilst in the former it was carried to its extremo intensification. Fluid forced backwards through the coronary trunks filled the muscular structure throughout, even to the columnse carneæ, by tributary veins reaching to the capillary system, and having some an arborescent, some a radiating, and others a parallel direction; but it did not fill a single vein either of the endo- or peri-cardium. On the other hand. fluid forced into the pericardiac trunks filled veins, even, in a case in which adhesions had formed, to the veins of the adhesive tissue, but did not enter into any branch of the coronary veins. The resuits appeared to show conclu sively that the venous system in the heart is arranged on the same plan as that in the lower limb, but that it cialminates in the direction-viz., from a state of free anas. tomosis between the sub- and super-fascial-the correlatives of the pericardiac and cardiac veins of the heart-in the foot, gradually upwards, through that of almost independency of each other in the thigh, of complete isolation in the heart.

Thus, with identity of textural elements, though varying in the relative proportions of each, there is also a striking degree of similarity in the plan on which the two vein systems in these apparently incongruous parts are respectively arranged.

But this vein arrangement, existing more or less through out the body, suggests a subdivision of veins into those which belong to the motor system, and, by inclusion, the organic, and those which belong to the tegumentary sub-dermoid; and, following this subdivision, there will appear to be other points of analogy between them which can materially assist in throwing light upon the paradox alluded to. Thus, from the comparative prox imity of the motor structures to the trunk vessels, it follows that a complete act of circulation through their textures is accomplished in a shorter period than that through the more distal tegumentary. And not only so, but the currents through the former (the motor) textures derive greater impulse from the action of the heart, as well as a larger supply of nutritive or oxidised bload Hence the tension of the blood in the motor veins has eves a tendency, as the radius of the circle it makes in the course of its circulation is less, to excess, and in all probability normally so. Further proof of this view is adduced from the fact that in the dead body blood injected into the femoral artery returns in the femoral vein before it appears in the capillaries of the skin; and that to fill these more forcible and frequently repeated strokes of the piston are required. The more highly carbonised and feebly flowing blood of the tegumentary system might probably afford some explantion of the excess in the deposit of the hydrocarbons amongst its textures-a reason which is supported by the anatomical correlative fact that the dermis pure 
has the advantage of returning its blood directly into the muscular or trunk system of veins, and only collaterally into the saphenous.

Again, the outflow of the blood from the two series of veins is, as $I$ have said, more advantageous to that of the motor veins. The points of confluence of the tegumentary with the systemic are by far more indirect courses, and in many instances at more acute angles than are those of the motor. Thus the coronary veins of the heart fall into the auricle; the pericardiac into the azygos. The internal jugular passes into the subclavian at a very easy angle, whilst the external passes through a powerful muscle and enters the same vein below it and at an acute angle. The large muscular veins of the arm go directly to the main stream, the cephalic and basilic veins to the axillary and subclavian; the azygos veins pass into the vena cava; the tegumentary into the vertebral, intercostal, lumbar, and sacral, and therefore, by indirect courses, into the systemic veins. Hence, also, why the motor veins are highly valved. The tegumentary veins have valves, but only to insure co-operation with those of the deep trunk veins, and to assist them in their functions; whilst in the veins of the organic system valves are for the most part wanting. The veins of the spinal cord go to the innominate, or to the vertebral, close to its junction with the innominate, and have valve cusps, like the tributaries of the external jugular, close to their termination, which is not the case with the terminal veins of their tegumentary allies. I might continue the illustration by reference to vein anatomy throughout the body. The main points are these: the veins of the motor and organic systems have short courses and direct access in their outflow to the deep trunk or systemic veins; their streams derive an exceptional amount of propulsive power from the heart, and, consequently, a tendency to increased blood-tension; whilst those of the tegumentary system have longer courses, indirect access for their streams to the trunks, and less blood-tention; hence, in case of any impediment to the passage of the blood through the main systemic veins, the streams in the organic and muscular vessels would have a preeminence in point of admission to the general circulation, whilst those of the tegumentary system would be exposed to proportionately exceptional difficulties.

From the foregoing facts and arguments, I think I may venture to claim some attention for the views which it is the object of this paper to set forth and elucidate-namely, (1) that the anatomy of the vein system, as well as a comparison of the histology of the subdermoid tegumentary connective tissue and the serous sac belonging to the heart, and inferentially to other serous sacs, indicate that they are analogous structures; (2) that if so, pericardial dropsy and anasarca or oedema are intrinsically analogous affections and due to the like direct causes; and (3) that the indirect cause lies in the physiological relation of the subdermoid to that of the muscular and organic vein circulation, in consequence of which retardation acting equally and coincidently on both would expose the former to different results -dropsy in the one, and, under certain conditions, parenchymatous congestion or inflammation in the other. ${ }^{1}$

The practical bearing of these conclusions has to do with exalted blood-tension in the systemic or deep trunk veins; and, in the former especially, with the question of relief by diet, by medicines calculated to lessen the volume of veinblood, but especially by bloodletting. This practice has undergone an entire change within the last quarter of a century or more, from a conventional habit, according to which persons judged for themselves, and were "blooded"

1 The analogies here referred to suggested to my mind the possibility of their being made to widen into a still more comprehensive and general idea-namely, that of a structural analogue between the heart and the limb. The only support I could find for such a Dadalean venture is from the works of Oken. In his curious work "Physico-Philosophy," he says" ${ }^{66}$ The blood in the human heart is the analogue of the bone in the limb since the muscles act upon it. If we take for the bone in the limb the os cordis found in the hearts of some vertebrates, and occasionally in man, and the great tendency to morbid deposits of bone, and earthy products in connexion with the endocardium, and the middle arterial coats, we may manufacture a quasi, if not a real, homology (structural) as follows :-

1. Fibrous envelope.

2. Pericardiam.

3. Fat.

4. Muscle.
5. Bone.

1. Dermis

2. Subcutaneous connective tissue and fascia.

3. Fat.

4. Muscle

5. Bone." on any embarrassment to the breatbing, and with, as I dis. tinctly remember, very marked benefit. Might it not be judiciously restored, and, under proper advice, become a source of like relief in cases of systemic blood-tension, indicated by dropsies and congestions of important organs, in our day? It is not for me to enlarge on this subject.

In the foregoing papers I have tried to indicate, by very feeble but sincere efforts, a mode by which, in my opinion, the study of the venous system might be renewed with a fair prospect of enlarging our knowledge of many subjects connected with our science that are at present avowedly but little understood, and of no department, perhaps, of that system with better earnest of success than its venocapillary.

Finsbury-place South.

\section{CASES OF "SUDDEN DEATH" AMONGST YOUNG CHILDREN.}

Bx J. PEARSON IRVINE, B.A., B.Sc., M.D., ASSISTAKT-PHYSICLAY TO CHABING.CROSS HOSPITAL.

THE cases recorded below are striking examples of what "sudden death" often means in quite young children. True sudden death is really rare amongst them, though the reports from coroner's courts seem to testify to the contrary. The cases also prove the uncertainty of the statements of friends, and the necessity of scientific investigation where there is the least douht. In these cages it is clear that had such investigation been declined verdicts at utter variance with the truth must have been recorded.

CASE 1.-A male child, aged eleven days, brought in dead. Autopsy seventy-two hours after death in moderately warm weather. The body was that of a well-formed child; external evidences of putrefaction not marked; post-mortem lividity in dependent parts; no discolouration of integuments of abdomen or thorax; on left cheek a bright-pink diffused flush, exactly like the hectic in phthisis, or the erythema of a superficial burn, and which gave place to deep lividity during the examination of the body; no excoriation, and no signs of violence in shape of bruises, wounds, or fractures. The brain was reduced to a softened pulps mass, and it was difficult to examine it even in situ. No pathological change could be detected either in it or its membranes. Be it observed here, that though putrefaction was so far advanced within the skull, evidences of it were elsewhere slight, the lungs being especially fresh. There was neither collapse, nor congestion of lungs, nor deformity of heart. The tongue was coated with a white fur, easily removed and catarrhal in nature. There was no hyperæmia of mouth or fauces. In the stomach was found about an ounce of meconium-like matter, afterwards discovered to consist in part of disintegrated blood. A limited fine capillary injection of the gastric mucous membrane near the pylorus, not hypostatic, and unaccompanied by post-mortem staining, but mixed up with punctated extravasations into the mucous membrane, to which the capillary injection was distinctly confined, was very remarkable. In the small intestine nothing was found; in the large the mucous membranes were more or less injected and punctated by extravasations, some of the latter being as large as a split-pea. In the cæcum these appearances were particularly decided. The contents of the large bowel were normally fæcal, and liver, kidneys, and spleen healthy.

An irritant was suspected, but no one in particular, and rough analysis of the contents of the stomach revealed nothing. The friends refused a history, maintaining that the child had died suddenly. But the evidence given at the inquest by Dr. Cantlie (the then resident medical officer in Charing-cross Hospital) led to an adjournment of the inquest, and to the acquirement of facts justifying the opinion formed on post-mortem examination, and throwing additional light on the cause of death. It was ascertained that to the child (aged eleven days) had been given a "cordial," which, after much care, Dr. Cantlie discovered to consist mainly of gin and aniseed. This had been administered, in what quantity could not, of course, be determined, with the intention, it was said, of "clearing away wind." It was proved during the adjourned inquiry that 